\title{
Symmetry-Induced Giant Vortex State in a Superconducting Pb Film with a Fivefold Penrose Array of Magnetic Pinning Centers
}

\author{
R. B. G. Kramer, A. V. Silhanek, J. Van de Vondel, B. Raes, and V. V. Moshchalkov \\ INPAC-Institute for Nanoscale Physics and Chemistry, Nanoscale Superconductivity and Magnetism Pulsed Fields Group, \\ K. U. Leuven, Celestijnenlaan 200 D, B-3001 Leuven, Belgium
}

(Received 23 March 2009; published 7 August 2009)

\begin{abstract}
A direct visualization of the flux distribution in a $\mathrm{Pb}$ film covering a fivefold Penrose array of Co dots is obtained by mapping the local field distribution with a scanning Hall probe microscope. We demonstrate that stable vortex configurations can be found for fields $H \sim 0.8 H_{1}, H_{1}$, and $1.6 H_{1}$, where $H_{1}$ corresponds to one flux quantum per pinning site. The vortex pattern at $0.8 H_{1}$ corresponds to one vacancy in one of the vertices of the thin tiles, whereas at $1.6 H_{1}$ the vortex structure can be associated with one interstitial vortex inside each thick tile. Strikingly, for $H=1.6 H_{1}$, interstitial and pinned vortices arrange themselves in ringlike structures ("vortex corrals") which favor the formation of a giant vortex state at their center.
\end{abstract}

Crystals are classified according to their translational and rotational symmetry. Until the mid 1980s, the lack of crystalline structures having a fivefold rotation symmetry axis was generally accepted. However, the revolutionary discovery in 1984 of crystals with "forbidden" symmetry [1] introduced a new family of crystallographic structures, known as quasicrystals, with order but not periodic. The existence of quasiperiodic arrays in two dimensions was earlier introduced by Penrose [2] in a mathematical context, who demonstrated that a two-dimensional plane can be fully covered by combining two different unit cells or tiles. The resulting structures possess long range orientational order without having long range translational order.

The interest in the unique properties of quasicrystals has nowadays surpassed the boundaries of the crystallographic community and reached most research fields in condensed matter physics [3-7]. Particular attention has been recently devoted to the pinning properties of flux lines in superconducting thin films with aperiodic, fractal, or quasiperiodic arrays of pinning sites [8-13]. In this case, the lack of translational order of the pinning landscape prevents the formation of one-dimensional channels for easy vortex flow, whereas the convolution of many build-in periods favors the proliferation of matching effects. In principle, both properties tend to improve the maximum current attainable without dissipation, i.e., the superconducting critical current.

Molecular dynamic simulations of driven vortices interacting with a fivefold two-dimensional Penrose array of pinning sites predicted local enhancements of the critical current for external fields $H=0.757 H_{1}, H_{1}$, and $1.482 H_{1}$, where $H_{1}$ is the field at which the density of singly quantized vortices coincides with the density of pinning centers $[8,9]$. The nontrivial matching features below and above $H_{1}$ should correspond to the occupancy of three out of four pinning sites in the vertices of the thin tiles and the presence of an interstitial vortex in each thick tile, respectively.
Indirect evidence of these stable vortex configurations has been obtained by transport measurements for a Penrose array of holes in a Nb film [11] and magnetic dots in $\mathrm{Al}$ and $\mathrm{Pb}$ films [12]. In both experimental investigations, further unforeseen features were reported. Unfortunately, the lack of direct visualization of the vortex distribution in this sort of system has concealed the real space vortex arrangement associated with the observed matching features in the transport properties.

In this work we establish a clear correlation between the theoretical predictions and the transport measurements by mapping the local field distribution in a $\mathrm{Pb}$ film covering a Penrose array of Co dots via scanning Hall probe microscopy (SHPM). The vortex patterns directly visualized at $H=0.765 H_{1}$ and $H=H_{1}$ are in agreement with the theoretical expectations. For $H>H_{1}$, interstitial vortices are placed inside the thick tiles, as anticipated by molecular dynamic simulations [8,9]. However, these interstitial vortices lie in a bistable state rather than in the geometrical center of the tile. Because of the long range vortex-vortex interaction, this degeneracy is lifted by the lack of reflection symmetry of the surrounding tiles. As a result, at certain magnetic fields the interstitial vortices accommodate themselves in order to achieve an ordered ringlike structure involving several tiles. Strikingly, this highly symmetric ringlike structure can stabilize a giant vortex state of two flux quanta at the center of the ring. This new symmetry-induced giant vortex state resembles that obtained in disk-shaped mesoscopic structures and results from the local field pressure exerted by the surrounding vortices. Complementary transport measurements show that this behavior leads to a clear matching feature at $H=$ $1.6 H_{1}$. Further unforeseen commensurability effects are also found at $H=3.8 H_{1}$ and $H=6.0 H_{1}$.

The investigated samples consist of a $50 \mathrm{~nm}$ thick $\mathrm{Pb}$ film evaporated directly on top of a fivefold Penrose array of square Co dots made by electron beam lithography and 
subsequent lift-off. The experimental procedure used for the sample preparation can be found in Ref. [14]. Figure 1 shows a scanning electron microscopy image of the dot array. For clarity, the magnetic particles have been connected by dotted lines to indicate the distribution of the thin and thick tiles, which represent the building blocks of the fivefold Penrose lattice. The size of the Co dots is $0.7 \mu \mathrm{m}$, and the length of the connecting lines is $3.1 \mu \mathrm{m}$. The pinning potential is created predominantly by a local depletion of superconductivity above the Co dots due to the proximity effect at the $\mathrm{Co} / \mathrm{Pb}$ interface. Experiments performed before and after magnetizing the dots show no differences, thus confirming that the electromagnetic coupling does not play a relevant role. For transport measurements, the samples were patterned in a bridge shape $102 \mu \mathrm{m}$ wide with a voltage contact separation of $392 \mu \mathrm{m}$. The $\mathrm{Pb}$ film has a critical temperature $T_{c 0}=$ $7.238 \mathrm{~K}$ as determined by $50 \%$ of the normal state resistance. A superconducting coherence length at zero temperature $\xi(0) \sim 33 \mathrm{~nm}$ was estimated by the slope of the superconducting/normal phase boundary of a reference film.

The scanning Hall probe microscopy images were obtained using a modified low temperature SHPM from Nanomagnetics Instruments [15]. Most of the SHPM images shown in this work were recorded at $4.2 \mathrm{~K}$ with a scanning area of $14 \times 14 \mu \mathrm{m}^{2}$ after field cooling the sample. Measurements performed at higher temperatures show no difference with those obtained at $4.2 \mathrm{~K}$, thus indicating that the vortex distribution is frozen at high temperatures close to the onset of the superconducting state. The images

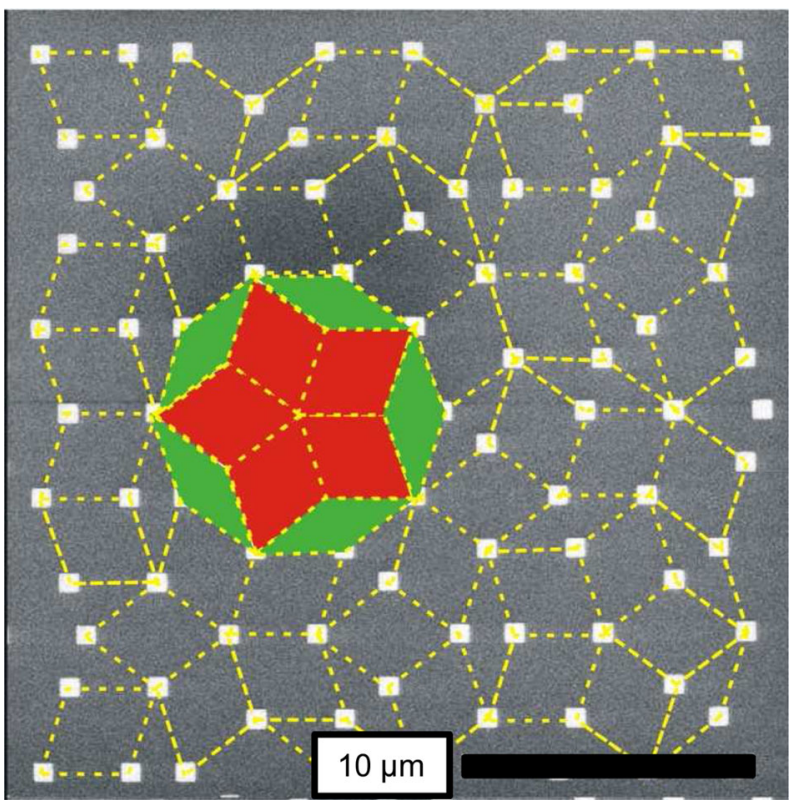

FIG. 1 (color online). Scanning electron microscopy image of the square dots distributed in a fivefold Penrose array. For clarity, the two types of tiles are indicated with dotted (yellow) lines. Five thin and thick tiles are painted with green and red, respectively. The black bar indicates a distance of $10 \mu \mathrm{m}$. were recorded in lift-off mode with the Hall sensor at about $500 \mathrm{~nm}$ above the surface of the sample. Homemade $x y$ positioners allow us to explore different regions of the same sample in order to avoid unwanted effects arising from the sample borders, imperfections, or small particles.

We first investigated the vortex dynamics of the superconducting sample by performing a series of currentvoltage characteristics as a function of field $H$ at temperatures very close to $T_{c 0}$. A detailed measurement of the voltage $V$ vs $H$ at constant current $I=300 \mu \mathrm{A}$ and $T=$ $7.21 \mathrm{~K}$ is shown in the upper panel in Fig. 2. The first matching field $H_{1} \sim 0.28 \mathrm{mT}$ can be clearly identified as a pronounced dip in the dissipation, indicating that each pinning site can trap a maximum of one flux quantum. An additional dip is present at $H \sim 0.76 H_{1}$ as predicted by molecular dynamic simulations. As we demonstrate below, this field corresponds to a vortex distribution leaving one vacancy in one of the vertices of the thin tiles.

To show the presence of stable vortex configurations at higher densities, we plot the depinning current $I_{c}$, using a dissipation criterion of $2 \mu \mathrm{V}$, for $T=7.1 \mathrm{~K}$ (Fig. 2, lower panel). Higher matching features can be seen for $H \sim$ $1.6 H_{1}, 3.8 H_{1}$, and $6.0 H_{1}$. These relatively high order matching features point out the relevance of the orientational order in stabilizing the vortex lattice. It is worth mentioning that typically commensurability effects in periodic pinning arrays are attributed to the perfect compensation of vortex currents, a condition which seems to be never satisfied in a quasiperiodic array.

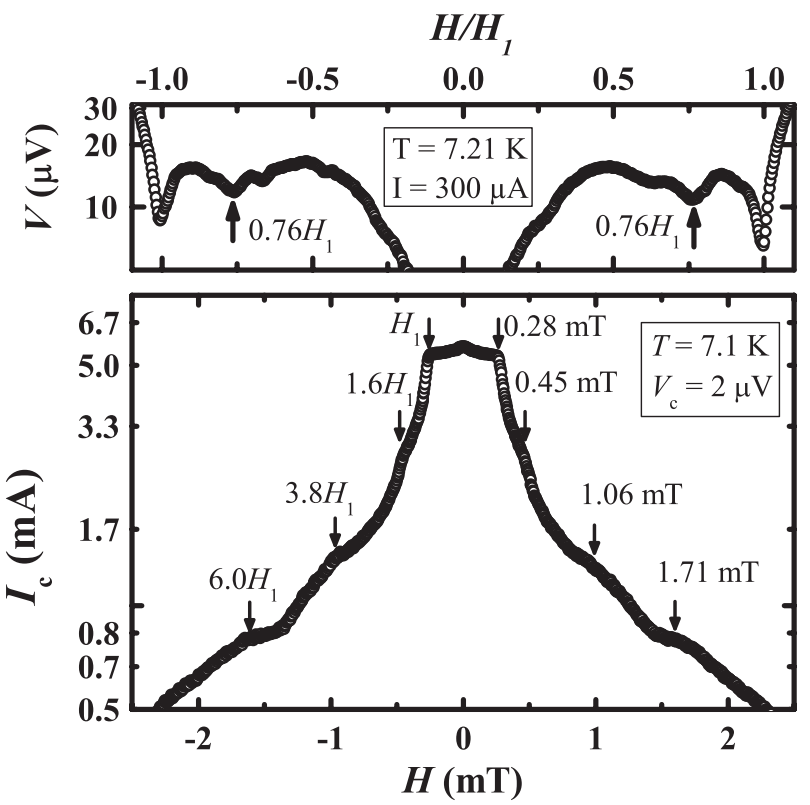

FIG. 2. The upper panel shows the voltage for a fixed bias current of $300 \mu \mathrm{V}$ as a function of the external field at $T=$ $7.21 \mathrm{~K}$. The lower panel shows the superconducting critical current, estimated with a voltage criterion of $2 \mu \mathrm{V}$, as a function of the external field for $T=7.1 \mathrm{~K}$. Matching features are indicated with black arrows. 
In order to clearly identify the microscopic vortex distribution associated with the different features observed in the $I_{c}(H)$ and $V(H)$ curves, we acquired scanning Hall probe microscopy images at different fields. A series of pictures obtained at $4.2 \mathrm{~K}$ after the field cooling procedure with fields ranging from -1 to $+1 \mathrm{mT}$ in steps of $0.005 \mathrm{mT}$ allowed us to determine the remanent field with high accuracy. Indeed, notice that steps of $0.010 \mathrm{mT}$ correspond to one extra vortex per scanning area between two consecutive images. Figure 3 summarizes the obtained images for a selected set of fields [16] (different columns) and at three different locations on the Penrose pattern (different rows). In this figure, we indicate the Penrose tiles with white lines for clarity. The determination of the dots' position was achieved by performing a scan in the presence of a large external field $(\sim 400 \mathrm{mT})$ which produces a clear contrast of the Co microparticles due to their magnetic moment strongly aligned by the perpendicular field.

The first column in Fig. 3 shows the local field profile obtained at $H \sim 0$. In this column, all panels show a single isolated vortex. The second column in Fig. 3 shows the vortex arrangement corresponding to the matching condition $H \sim 0.76 H_{1}$ (see also Fig. 2). It is clear from this image that unoccupied pinning sites are located at one of the vertices of the thin tiles. This vortex distribution was anticipated by Misko, Savel'ev, and Nori [9] based on the rapid increase of the vortex repulsion as the vortex separation decreases. The third column in Fig. 3 corresponds to the exact commensurability of the vortex lattice with the

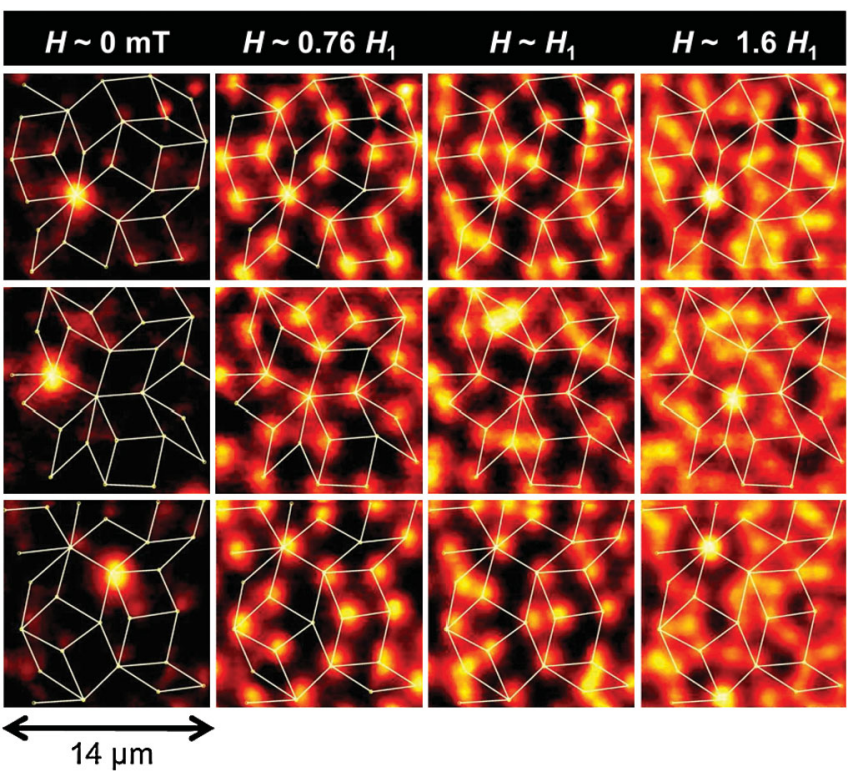

FIG. 3 (color). Scanning Hall probe microscopy images obtained after field cooling the sample down to $4.2 \mathrm{~K}$. Each row corresponds to a different location of the array. The first, second, third, and fourth columns correspond to $H \sim$ $0 \mathrm{mT}, H \sim 0.220 \mathrm{mT} \sim 0.76 H_{1}, H \sim 0.275 \mathrm{mT} \sim H_{1}$, and $H \sim$ $0.455 \mathrm{mT} \sim 1.6 H_{1}$, respectively. pinning landscape; i.e., every vortex sits on top of a Co dot. The last column illustrates the vortex pattern obtained at $H \sim 1.6 H_{1}$. Here, due to the repulsive interaction between vortices, interstitial vortices sit only inside the thick tiles. Differential images, obtained by subtracting two consecutive field coolings, show that these interstitial vortices are very mobile and tend to avoid the geometrical center of the tile [Fig. 4(c)].

This finding indicates that an interstitial bistable configuration takes place inside each thick tile. The formation of ringlike structures or vortex corrals clearly seen in the last column of Fig. 3 demonstrates that a long range interaction eventually lifts the twofold degeneracy of the interstitial positions. More importantly, a detailed analysis of the field intensity at the core of the ring-shaped vortex structures indicates that the central vortex carries two flux quanta $\phi_{0}$ [see Fig. 4(f)]. These multiquanta vortices are not a mere consequence of stochastic distribution of pinning strength since they appear at well defined locations on the pinning landscape corresponding to multinodal agglomeration of Penrose tiles.

To obtain a better insight into the mechanism responsible for the creation of these multiquanta vortices, we show the field profile corresponding to two consecutive images and their difference [Figs. 4(a)-4(c)] exactly at the creation of a vortex corral $\left(H \sim 1.6 H_{1}\right)$. A reconfiguration

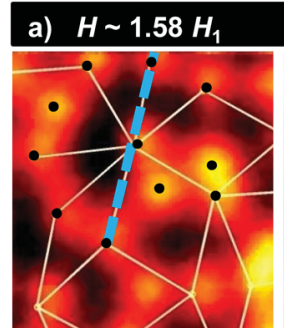

d) $H \sim 1.58 H_{1}$

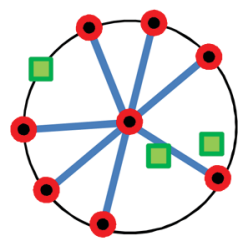

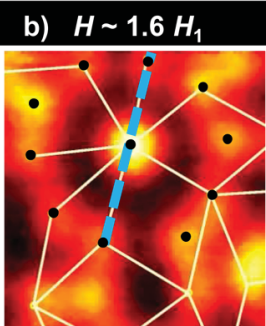

e) $H \sim 1.6 H_{1}$

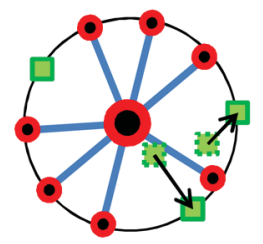

c) difference

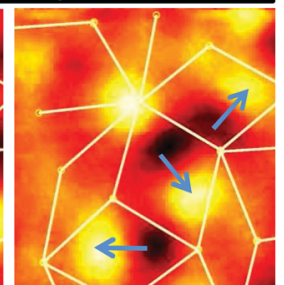

f) field profile

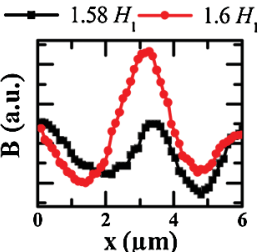

FIG. 4 (color). Scanning Hall probe microscopy images obtained at two consecutive field values (a) $H \sim 0.445 \mathrm{mT} \sim$ $1.58 H_{1}$ and (b) $H \sim 0.45 \mathrm{mT} \sim 1.6 H_{1}$ together with (c) the differential image taken at a specific location where the vortex corral appears. Black dots in (a) and (b) mark the vortices illustrated in (d) and (e). Black and white spots in (c) indicate the change of position of individual vortices. In (d) and (e), a schematic representation of the vortex distribution is given for the two consecutive field values. Red circles represent pinned vortices, and green squares represent the interstitial vortices. (f) shows the magnetic field profile obtained along the dotted line drawn in (a) and (b) for both magnetic field values. The integrated field for $1.6 H_{1}$ clearly corresponds to the giant $2 \phi_{0}$ vortex. 
of the interstitial vortices creates a highly symmetrical vortex structure around specific multinodal points of the Penrose lattice. This process is schematically presented in Figs. 4(d) and 4(e), which show the distribution of pinned (red circles) and interstitial (green squares) vortices for both consecutive magnetic fields. At $H \sim 1.6 H_{1}$, the mobile interstitial vortices rearrange themselves in a structure with high rotational order. The exact locations, where the vortex corral appears, are surrounded by ten vortices, seven pinned at the Penrose lattice and three interstitial vortices. As a result, a tenfold rotational symmetry is obtained [Figs. 4(b) and 4(e)]. At the center of this highly symmetric vortex structure, a multiquanta vortex is favored.

This unexpected result resembles the symmetry-induced giant vortex states in mesoscopic or microstructured superconductors close to the superconducting/normal phase boundary [17-21]. In that case, the boundary conditions impose the sample symmetry on the order parameter which manifests itself in the formation of unconventional vortex patterns as giant vortices or vortex-antivortex pairs. Here the combination of the applied magnetic pressure, induced by the surrounding vortices, and the high local symmetry leads to the generation of a multiquanta vortex at the center of the vortex corral. This is in agreement with previous experimental [17,22] and theoretical [23] reports showing that the number of vortices trapped by a single pinning site increases with increasing external field. In our particular case, it is the highly inhomogeneous local field which drives the system to the multiquanta vortex state.

It is important to emphasize that the theoretical investigations of the vortex matter in a fivefold Penrose array performed so far $[8,9]$ assumed a short range interaction between vortices. Since the present work has been done on a thin $\mathrm{Pb}$ film with thickness smaller than the temperaturedependent penetration depth, a more suitable comparison should be done with long range repulsive Pearl vortices in the presence of a quasiperiodic array of pinning sites [24]. The combination of this long range vortex-vortex interaction with the large penetration depth expected by freezing the flux lattice at high temperatures is most likely a necessary ingredient for the formation of a giant vortex state at $H \sim 1.6 H_{1}$.

In conclusion, we have investigated the vortex distribution in a superconducting film with a Penrose lattice of Co dots by scanning Hall probe microscopy. The obtained images confirmed the theoretically predicted vortex patterns at low densities (i.e., $H \leq H_{1}$ ). A much richer vortex structure is found above $H_{1}$, where mobile interstitial vortices create highly symmetrical vortex corrals around particular pinning sites of the Penrose lattice. The formation of vortex rings in combination with long range vortexvortex interaction leads to the stabilization of multiquanta vortices at the core of these rings.

This work was supported by Methusalem Funding of the Flemish government, FWO-Vlaanderen, the Belgian Inter-
University Attraction Poles IAP, and the ESF-NES Programs. A. V. S. and J. V. d. V are grateful for the support from the FWO-Vlaanderen.

[1] D. Shechtman, I. Blech, D. Gratias, and J. W. Cahn, Phys. Rev. Lett. 53, 1951 (1984).

[2] R. Penrose, Bull. Inst. Math. Appl. 10, 266 (1974).

[3] F. Nori and J.P. Rodriguez, Phys. Rev. B 34, 2207 (1986).

[4] M. Kohmoto, B. Sutherland, and C. Tang, Phys. Rev. B 35, 1020 (1987).

[5] K. N. Springer and D. J. Van Harlingen, Phys. Rev. B 36, 7273 (1987).

[6] D. R. Nelson and B. I. Halperin, Phys. Rev. B 19, 2457 (1979).

[7] E. Abe, Y. Yan, and S. J. Pennycook, Nature Mater. 3, 759 (2004).

[8] V. Misko, S. Savel'ev, and F. Nori, Phys. Rev. Lett. 95, 177007 (2005).

[9] V. R. Misko, S. Savel'ev, and F. Nori, Phys. Rev. B 74, 024522 (2006).

[10] J. E. Villegas, M. I. Montero, C.-P. Li, and I. K. Schuller, Phys. Rev. Lett. 97, 027002 (2006).

[11] M. Kemmler, C. Gürlich, A. Sterck, H. Pöhler, M. Neuhaus, M. Siegel, R. Kleiner, and D. Koelle, Phys. Rev. Lett. 97, 147003 (2006).

[12] A. V. Silhanek, W. Gillijns, V. V. Moshchalkov, B. Y. Zhu, J. Moonens, and L. H. A. Leunissen, Appl. Phys. Lett. 89, 152507 (2006).

[13] C. Reichhardt and C. J. Olson Reichhardt, Phys. Rev. B 76, 094512 (2007).

[14] S. Raedts, A. V. Silhanek, V. V. Moshchalkov, J. Moonens, and L.H.A. Leunissen, Phys. Rev. B 73, 174514 (2006).

[15] S. J. Bending, Adv. Phys. 48, 449 (1999).

[16] It is worth emphasizing that more than 600 images were acquired at each of the three different spots. See EPAPS Document No. E-PRLTAO-103-037934 for an illustrative movie showing the evolution of the vortex pattern. For more information on EPAPS, see http://www.aip.org/ pubservs/epaps.html.

[17] V. V. Moshchalkov, X. G. Qiu, and V. Bruyndoncx, Phys. Rev. B 55, 11793 (1997).

[18] V. Bruyndoncx, J. G. Rodrigo, T. Puig, L. Van Look, V. V. Moshchalkov, and R. Jonckheere, Phys. Rev. B 60, 4285 (1999).

[19] A. Kanda, B. J. Baelus, F. M. Peeters, K. Kadowaki, and Y. Ootuka, Phys. Rev. Lett. 93, 257002 (2004).

[20] B. J. Baelus, L. R.E. Cabral, and F. M. Peeters, Phys. Rev. B 69, 064506 (2004).

[21] G. R. Berdiyorov, M. V. Milosevic, and F. M. Peeters, Phys. Rev. Lett. 96, 207001 (2006).

[22] A. Bezryadin, Yu. N. Ovchinnikov, and B. Pannetier, Phys. Rev. B 53, 8553 (1996).

[23] M. M. Doria and G. F. Zebende, Phys. Rev. B 66, 064519 (2002).

[24] J. Pearl, J. Appl. Phys. 37, 4139 (1966). 